\title{
SAFETY AND EFFICACY OF STEM CELL THERAPY FOR TREATMENT SEVERE TRAUMATIC BRAIN INJURY
}

\author{
Nguyen Viet Quang Hien*, Le Hoang Duy Minh ${ }^{\star \star \star}$, Phan Thi Dieu Ngan***, \\ Le Gia Vinh**, Nguyen Viet Quang*, Phan Thi Thuy Hoa*, Che Thi Cam Ha***
}

\section{ABSTRACT}

Traumatic brain injury (TBI) is a complicated topic.TBI has expensive treatment, sequelae, high mortality and efficient therapeutic strategies for TBI are urgently needed. Patients with severe TBI account for $10 \%$ of all traumatic brain injury patients, but more than $80 \%$ of patients who die from traumatic brain injury are in this group of patients. That proves the danger of severe TBI. Mesenchymal stem cells (MSCs) are multipotent cells with high proliferative and self-renewal capacities, as well as immunomodulatory and neuroregenerative effects. MSCshave been shown to treat TBI and significantly reduce inflammation of injured tissue. In the present study, we evaluated the quality and effectiveness of mesenchymal stem cell transplantation with the graft dose of $9 \times 10^{6} \mathrm{cells} / \mathrm{kg}$. Clinical indexes (Pulse, temperature, SBP, HATTr, HATTB, GCS) and subclinical (Hepatic index, kidney, cortisol, lactate, glucose, hematological index, respiratory index) were monitored on 60 patients within 6 months.

The results showed that the recovery journey is different for every person with the graft dose of $6-9 \times 10^{6}$ cells $/ \mathrm{kg}$, but there were no adverse complications for the patients, the clinical and paraclinical indicators were stable. No significant side effects were observed during the trial as well as after 6 months of treatment. The MSC transfusions significantly increased the life

\footnotetext{
* Hue Central Hospital

** Vietnam Medical Association

*** Hue University
}

Responsible person: Che Thi Cam Ha

Email: chethicamha@husc.edu.vn

Date of receipt: $05 / 7 / 2021$

Date of scientific judgment: 01/8/2021

Reviewed date: 23/8/2021 quality patients; reduced brain damage and increased HSC counts. Inflammatory cytokines (IL-6) levels decreased and GCS increased in the MSC transfusion group, indicating that the patient's health improved.

Keywords: Traumatic brain injury, mesenchymal stem cell, mononuclear cells, transplantation, neuroimmunity, antiinflammation

\section{INTRODUCTION}

Traumatic brain injury (TBI) is a leading cause of death and disability, affecting approximately 10 million people worldwide each year. Among all types of trauma, brain trauma that is most likely to result in death or permanent disability, high or low grade both causes profound and long-lasting damage to the patient. Even mild TBI can lead to persistent symptoms and serious long-term sequelae. Although trauma management systems and surgical techniques have improved, the overall mortality in severe TBI remains $\sim 25 \%$. Patients often complain of headaches for weeks after the injury and are likely to face long-term effects such as depression, Alzheimer's, or senile dementia, affecting their families and communities of patients [1]. Therefore, rehabilitation therapy is important to maximize rehabilitation after TBI. However, there are still limitations in current treatments because of the heterogeneity of injury based on location and extent of lesions, as well as patient age and 
comorbidities, pose a significant challenge in the development of effective treatments for TBI [2].

Surgery is often necessary for patients with more serious injuries. However, to date, there are no therapies that completely resolve the brain damage suffered following the trauma. Stem cells, due to their capacity to differentiate into neuronal cells and through releasing neurotrophic factors, seem to be a valid strategy to use in the treatment of traumatic brain injury. Mesenchymal stem cells (MSCs) are a promising strategy for the treatment of TBI. MSCs isolated from bone marrow have the ability to self-renew and differentiate into bone, cartilage, fatand nerve cells [3]. They participate in normal homeostasis and confer endogenous or posttransplant therapeutic benefits of organs, i.e. brain[4]. Furthermore, MSCs have homing capacity, which is a favorable mechanism for introducing stem cells into delicate sites such as in the brain [5]. Studies around the world have shown the safety of stem cell transplantation for patients.[6].[7]

Bone marrow derived MSCs have been considered for the treatment of different diseases.Autologous MSCs carry a safer pattern without the risk of malignant transformation. In the present study, we analyzed the subclinical and clinical parameters of 30 patients receiving mononuclear and stem cell transplantation. From there, evaluate the effectiveness of stem cell transplantation dose as well as its safety in the treatment of severe TBI.

\section{PATIENTS AND METHOD}

\section{Patient selection criteria}

These studies were approved by the ethic committee of Hue Central Hospital in Vietnam. All the participants or the next of kin, caretakers, or guardians provided their written informed consent to participate in this study. Participating patients were divided into 2 groups including control group and MSC group. Patient outcome evaluation.

All patients were assessed by a protocol, including comprehensive physical and neurological examination, routine testing, hematological index before the procedure and after transplantation. The neurological outcome of patients was evaluated via Glasgow Coma Scale, National Institute of Health Stroke Scale (NIHSS), and Barthel index (BI) [6-8].

In MSC group, there are 30 patients, aged 14 - 56 years, weighing $45-83 \mathrm{~kg}$, hospitalized in a coma and treated with routine methods during and after the study of combining stem cell transplantation. The control group included 30 patients, aged 1863 , weighing $45-75 \mathrm{~kg}$, hospitalized in a coma and treated with routine methods. The patients were formed according to the Glasgow score (GCS). The lower GCS score indicates the more severe disease and the higher the risk of death.

The lower GCS score indicates more severe disease and higher risk of death, which the lowest point is 1 while the highest point is 4-6 and the total added score of all test ranges from 3 to 15 . Patients with GCS $\leq 8$ scores are considered to have severe brain damage and patients with GCS> 8 scores are considered to have moderate brain damage. Patients were excluded if they met any of the following criteria: i) a participant in the study 
required a blood transfusion, no sample was taken from that patient to check hematological indicators; ii) TBI was combined with other internal organ injuries, history or family with peripheral neuropathy or autoimmune disease; iii) Cognitive limitations, chronic inflammatory diseases, malignant neoplasms, chronic liver disease; iv) Regular anti-inflammatory drug use, blood pathology or respiratory instability; v) Pregnant or possibly pregnant; and vi) Patients with cancer or malignant diseases

\section{Bone Marrow Harvest}

The bone marrow collection procedure was carried out under sterile conditions. The bone marrow was aspirated from the anterior pelvic position. Doctors conducted 3-6 aspirate positions on the pelvis to ensure enough quantity of cells for transplantation. The bone marrow volume could be harvested up to $150 \mathrm{~mL}$ buffy coat. Blood pressure, heart rate, oxygen saturation in the vessels, and meningeal pressure were checked every 5 minutes within 1 hour.

\section{Stem cell characterization engraftment criteria}

Adipogenic, chondrogenic and and osteogenicdifferentiation of MSCs: For MSC characterization, adipogenic, chondrogenic and osteogenic differentiation assays were performed on isolated cells for all patients. Alizarin Red S, Oil Red O and Alcian Blue $1 \%$ (Miltenyi Biotec, Germany) were staining to analyze osteogenic, adipogenic and chondrogenic lineages, respectively.

MSCs must be assessed for their ability to form Colony-forming unit - fibroblasts (CFU-Fs). MNCs were cultured in T25-flask, the density of $3 \times 10^{4}$ cells $/ \mathrm{cm}^{2}$. After 10-14 days, the cell clusters were stained with Giemsa. The CFU-F clusters were defined as a cluster with a diameter of about 1-8 mm with more than 50 cells.

The ratio of living cells should be higher than $95 \%$, with the ability to create CFU-F clusters. The percentage of marker for MSCs should be higher than $95 \%$ and positive for differentiation into three lineages.

Cell Product Infusion

MNCs transplantation for the first time was roundly 2-3 hours after bone marrow aspiration; the cells were transplanted into a patient through intravenous injection. The 2nd transplantation was performed after harvesting enough MSCs, ranging 14-20 days. Post-infusion monitoring of postharvest hemodynamics was performed for 6 hours. The control group was treated by conventional medicine, including Mannitol, Rabeprazole and Cephalosporin, Carbapenem and Vancomycin antibiotics.

\section{Statistical Analysis}

Data were expressed as the Mean \pm SEM with the GraphPad Prism 8 software (LLC, San Diego, USA). Differences were determined by using Student's t-test, *P < $0.05, * * \mathrm{P}<0.01$ between two groups.

\section{RESULTS}

\section{Patient}

Inflammation and swelling inside the brain often damage nerves and prevent areas of the brain from receiving the oxygen and glucose it needs to perform. These effects of the injury disrupt the normal function of the brain. 


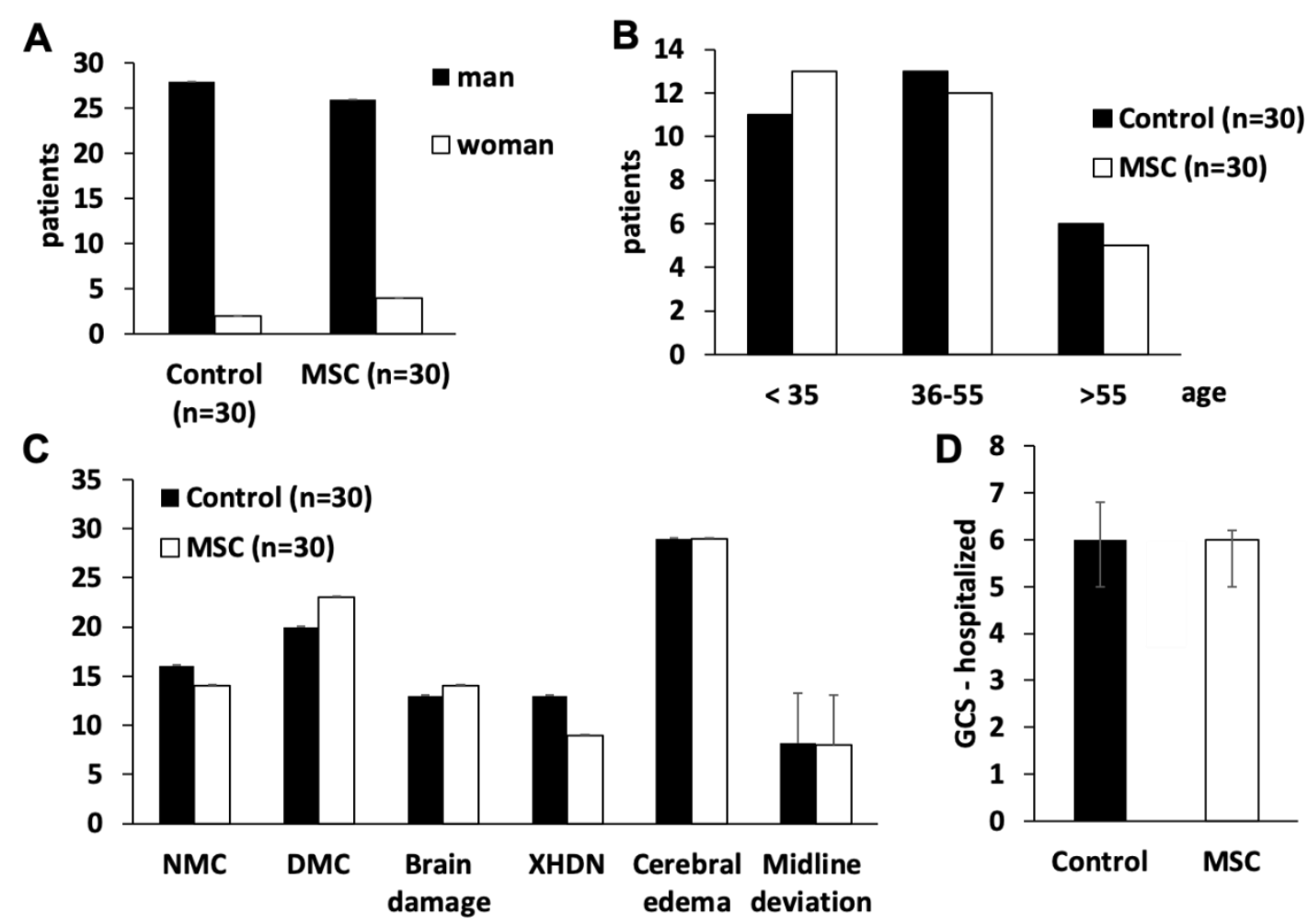

Figure 1. Selected patients to participate in the research

The results of disease selection showed that, there was no big difference between the sex and age brackets between the control and cell transplant groups (Fig. 1A, B). The patients were all hospitalized with severe TBI with a GCS index of $6.00 \pm 0.8$ in the control group and $6.00 \pm 0.2$ in the transplant group (Fig. 1.C, D)

\section{Quality and stem cell transplantation}

Table 1. Stem cell quality and graft dose

\begin{tabular}{|c|c|}
\hline Amount of bone marrow & $260 \pm 30 \mathrm{ml}$ \\
\hline monocyte survival rate & $98,56 \%$ \\
\hline Mesenchymal cell transplantation time & $15 \pm 3$ (days) \\
\hline Mesenchymal cell survival rate & $99 \%$ \\
\hline Mycoplasma & Âm tính \\
\hline Mono cells (MNC) & $3-6 \times 10^{6} \mathrm{cell} / \mathrm{kg}$ \\
\hline Mesenchymal stemcells(MSC) & $6-9 \times 10^{6} \mathrm{cell} / \mathrm{kg}$ \\
\hline
\end{tabular}

The results of the isolation and culture process showed that the quality of cells for transplantation of MNCs and MSCs ensured low endotoxin concentrations, negative for microorganisms and mycoplasma, and high cell viability (Table 1). In the present study, to evaluate the effectiveness of the study, we used a dose of MNC and MSC, both $6-9 \times 10^{6}$ cells $/ \mathrm{kg}$. 

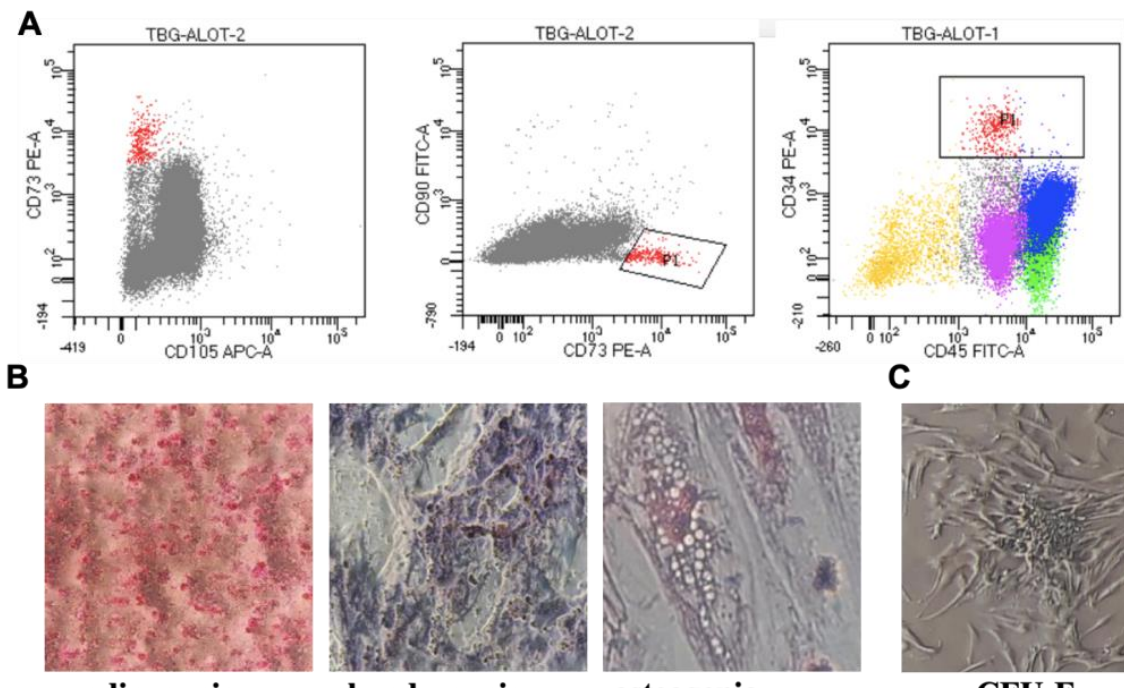

C

adipogenic

chondrogenic

osteogenic

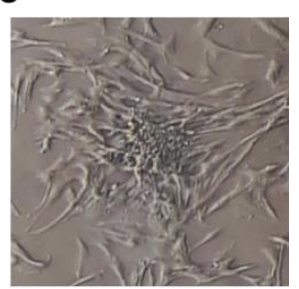

CFU-F

Figure 2. Stem cell characterization, engraftment criteria and evaluation of cell quality

FACS results showed that MNCs cells were negative for CD45, CD34 (1-2\% positive) (Figure 2A). For MSCs, the results showed positivity for CD73, CD90, CD105 (96-98\%) after culture (Figure 2A). All MSCs with clustering ability (Figure 2C) were successfully differentiated into adipogenic, osteogenic, and chondrogenic lines (Figure 2B). The farming process time ranges from 10-16 days depending on the age and the number of harvested MNC.

\section{Clinical and subclinical results after 6 months of treatment}

Patients after MNC and MSC transplantation were followed up continuously for 6 months

\subsection{Clinical results}

Table 2. Hematological index in 6 months

\begin{tabular}{|c|c|c|c|c|c|c|c|c|c|c|}
\hline & \multirow{2}{*}{\multicolumn{2}{|c|}{ Mono cell }} & \multicolumn{8}{|c|}{ MSC } \\
\hline & & & \multicolumn{2}{|c|}{15 day } & \multicolumn{2}{|c|}{1 month } & \multicolumn{2}{|c|}{3 months } & \multicolumn{2}{|c|}{6 months } \\
\hline & $\begin{array}{l}\text { Control } \\
(n=30)\end{array}$ & $\begin{array}{c}\text { Mono } \\
(\mathrm{n}=30)\end{array}$ & $\begin{array}{l}\text { Control } \\
(n=27)\end{array}$ & $\begin{array}{c}\text { MSC } \\
(n=28)\end{array}$ & $\begin{array}{l}\text { Control } \\
(n=22)\end{array}$ & $\begin{array}{c}\text { MSC } \\
(n=23)\end{array}$ & $\begin{array}{l}\text { Control } \\
(n=21)\end{array}$ & $\begin{array}{c}\text { MSC } \\
(n=23)\end{array}$ & $\begin{array}{l}\text { Control } \\
(n=20)\end{array}$ & $\begin{array}{c}\text { MSC } \\
(n=22)\end{array}$ \\
\hline Pulse & $\begin{array}{c}73,80 \\
\pm \\
10,90\end{array}$ & $\begin{array}{c}79,90 \\
\pm \\
17,00\end{array}$ & $\begin{array}{c}72,60 \\
\pm \\
10,90\end{array}$ & $\begin{array}{c}77,60 \\
\pm \\
16,08\end{array}$ & $\begin{array}{r}74,00 \\
\pm 8,61\end{array}$ & $\begin{array}{c}75,14 \\
\pm \\
17,28\end{array}$ & $\begin{array}{c}85,13 \\
\pm 7,57\end{array}$ & $\begin{array}{c}78,33 \\
\pm \\
14,31\end{array}$ & $\begin{array}{c}78,33 \\
\pm \\
14,31\end{array}$ & $\begin{array}{c}71,80 \\
\pm \\
15,09\end{array}$ \\
\hline Temp & $\begin{array}{r}37,40 \\
\pm 0,37\end{array}$ & $\begin{array}{r}37,40 \\
\pm 0,44\end{array}$ & $\begin{array}{r}37,60 \\
\pm 0,45\end{array}$ & $\begin{array}{r}37,90 \\
\pm 0,84\end{array}$ & $\begin{array}{c}37,80 \\
\pm 0,75\end{array}$ & $\begin{array}{r}38,30 \\
\pm 0,84\end{array}$ & $\begin{array}{c}37,50 \\
\pm 0,45\end{array}$ & $\begin{array}{c}37,77 \\
\pm 0,75\end{array}$ & $\begin{array}{c}37,77 \\
\pm 0,75\end{array}$ & $\begin{array}{c}37,12 \\
\pm 0,66\end{array}$ \\
\hline HATT & $\begin{array}{c}106,00 \\
\pm \\
17,00 \\
\end{array}$ & $\begin{array}{c}111,00 \\
\pm \\
31,00 \\
\end{array}$ & $\begin{array}{c}115,20 \\
\pm \\
17,00 \\
\end{array}$ & $\begin{array}{c}117,00 \\
\pm \\
26,00 \\
\end{array}$ & $\begin{array}{c}113,22 \\
\pm \\
15,20 \\
\end{array}$ & $\begin{array}{c}115,40 \\
\pm \\
22,00 \\
\end{array}$ & $\begin{array}{c}106,34 \\
\pm \\
12,12 \\
\end{array}$ & $\begin{array}{c}118,42 \\
\pm \\
12,40 \\
\end{array}$ & $\begin{array}{c}118,42 \\
\pm \\
12,40\end{array}$ & $\begin{array}{c}115,24 \\
\pm \\
10,20 \\
\end{array}$ \\
\hline HATTr & $\begin{array}{c}64,20 \\
\pm \\
13,90\end{array}$ & $\begin{array}{c}66,00 \\
\pm \\
11,20 \\
\end{array}$ & $\begin{array}{c}70,14 \\
\pm \\
13,90 \\
\end{array}$ & $\begin{array}{c}62,00 \\
\pm 8,20\end{array}$ & $\begin{array}{c}72,06 \\
\pm \\
10,56 \\
\end{array}$ & $\begin{array}{r}65,60 \\
\pm 7,24\end{array}$ & $\begin{array}{c}75,44 \\
\pm \\
10,00\end{array}$ & $\begin{array}{c}68,68 \\
\pm 8,15\end{array}$ & $\begin{array}{r}68,68 \\
\pm 8,15\end{array}$ & $\begin{array}{l}66,33 \\
\pm 7,81\end{array}$ \\
\hline HATB & $\begin{array}{c}78,00 \\
\pm \\
14,00 \\
\end{array}$ & $\begin{array}{c}81,20 \\
\pm \\
15,20\end{array}$ & $\begin{array}{c}75,00 \\
\pm \\
12,00 \\
\end{array}$ & $\begin{array}{c}88,70 \\
\pm \\
14,32 \\
\end{array}$ & $\begin{array}{c}72,06 \\
\pm \\
10,00 \\
\end{array}$ & $\begin{array}{c}84,40 \\
\pm \\
12,20 \\
\end{array}$ & $\begin{array}{r}70,14 \\
\pm 9,59\end{array}$ & $\begin{array}{r}82,20 \\
\pm 8,31\end{array}$ & $\begin{array}{r}82,20 \\
\pm 8,31\end{array}$ & $\begin{array}{r}79,44 \\
\pm 6,42\end{array}$ \\
\hline
\end{tabular}


A

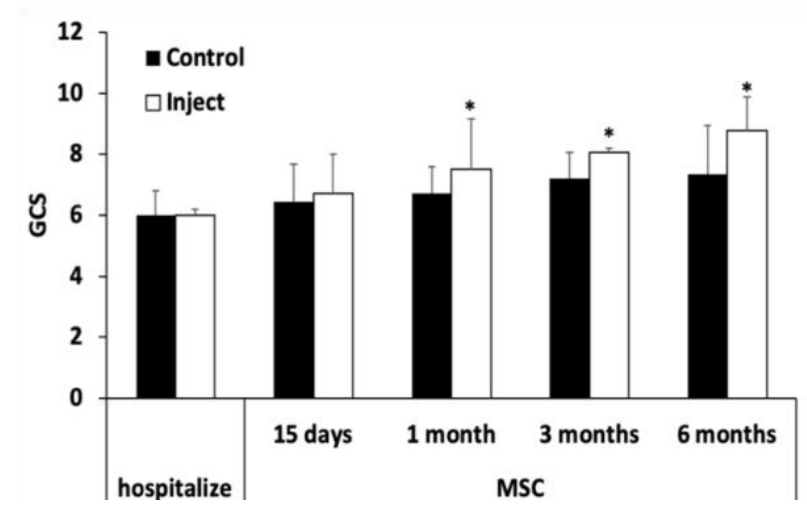

B

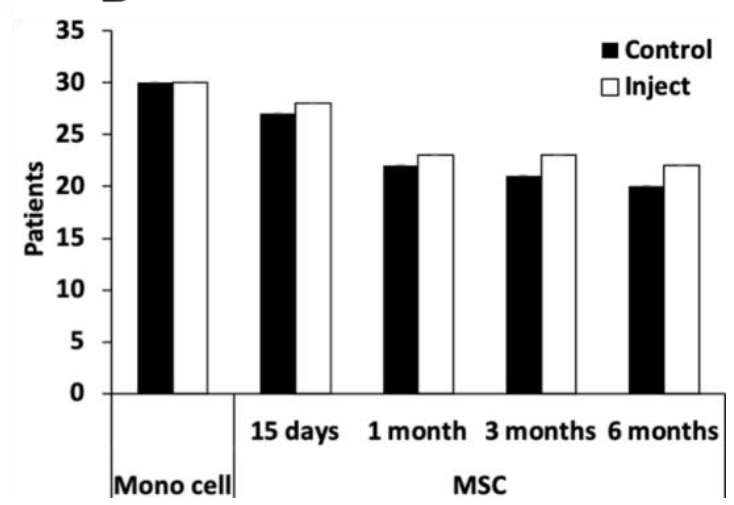

Figure 3. GCS index and number of patients after 6 months

Some studies demonstrated axonal neuroprotection after MSCs therapywhich is probably conducted via the synthesis of neurotrophic factors, and also immunomodulatory properties of MSCs.Clinical results showed that after stem cell transplantation, the patient's survival indicators, including pulse, temperature, BP, HATTr, HATTB had no difference compared with the control group. These results were similar at 1 month, 3 months, and 6 months of follow-up with no difference between the 2 groups (Table 2).In total, there were 18 deaths (Control: 10 and MSC: 8), the cause of death was not caused by complications of MSCs (Figure 3B). This shows that with the graft dose of 9x106 cells $/ \mathrm{kg}$ in MNC and MSC, there is a high safety in the treatment of MSCs.

In contrast, GCS index changed between the 2 study groups, and the difference was evident after 1 month of stem cell transplantation (Control: $6.70 \pm 0.89$, MSC: $7.50 \pm 1.65$ ). , according to the GCS scale 912 classified as a moderate injury (Figure
3A) [8]. A total of 18 deaths (Control: 10 and MSC: 8) were reported, the cause of death was not caused by complications of MSC.Although full efficacy results have not been investigated underlying cellular and molecular mechanism. The result of the experiment show that intravenous administration MSCs are able to traffic into the brain lesions and improve the survival rate of brain cells. It is also shown that MSCs transplantation significantly improves the clinical.Student t-tests calculate the significant difference between two groups, $* * \mathrm{P}<0.01 ; * \mathrm{P}<0.05$.

\subsection{Monitor inflammatory responses} result

The test measures the amount of liver, kidney, IL-6, cortisol, lactate, glucosein the blood.A blood sample drawn from a vein in patients arm, commonly ordered signs and symptoms associated with one of the conditions treatment TBI before and after stem cell transplantation. 


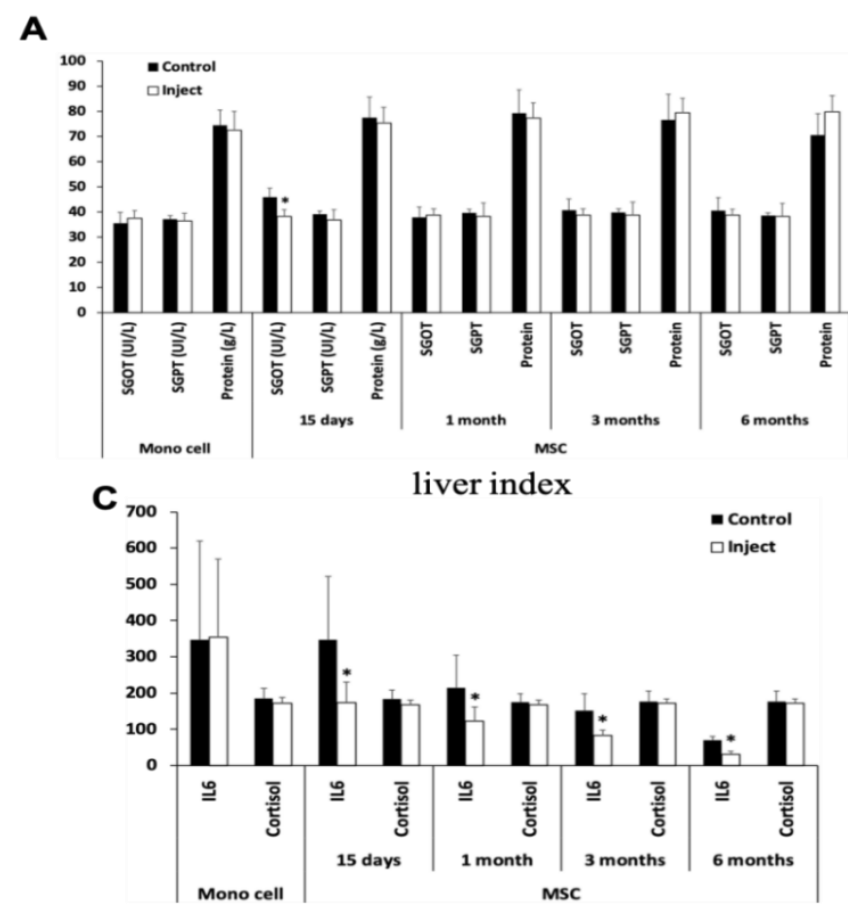

IL6, Cortisol

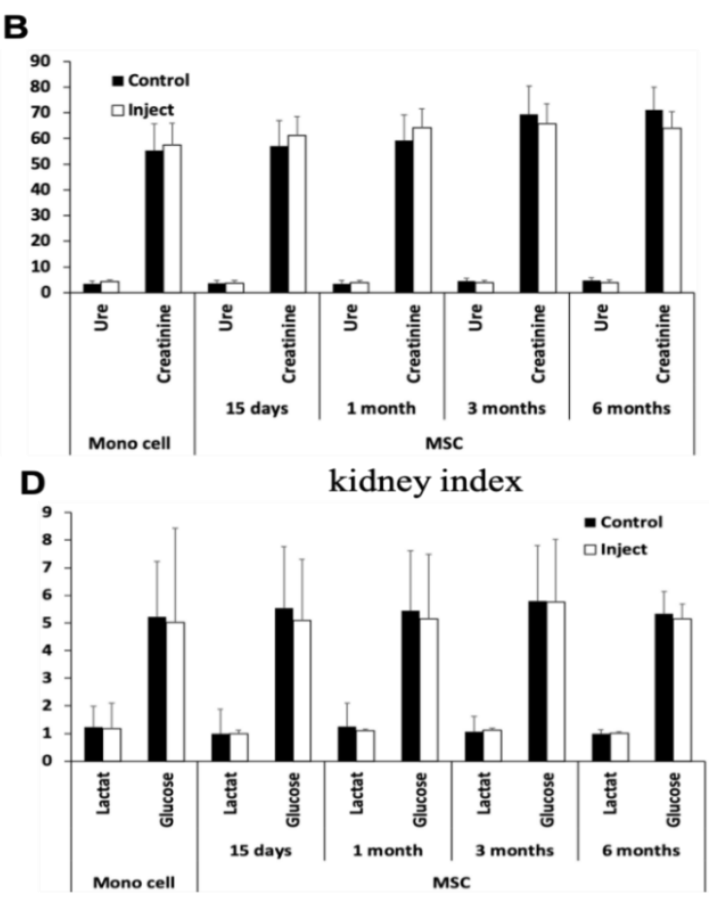

Lactat, Glucose

Figure 4. Index of Liver, kidney, IL-6, Cortisol, Lactate, Glucose.

Cytokines have multiple roles to play within the body and act especially within the immune system to help direct the body's immune response. IL-6 also plays a role in body temperature regulation, bone maintenance, and brain function. It is primarily pro-inflammatory but can also have anti-inflammatory effects.Plasma concentrations of IL-6 in the control group and MSC group were determined before treatment and after 6 hours, 12 hours, 24 hours, 72 hours, one week, one month, 3 months and 6 months after treatment. The concentrations of IL-6 (291.75 pg/mL) in control subjects were significantly increased to the peak at 24 or $72 \mathrm{~h}$ before decrease afterward.

However, these cytokines and proteins' levels were still higher than the range of normal indices. In the MSC group, IL-6 levels after TBI were significantly ameliorated than those of control subjects at 6 hours - 2 months following injury.

The concentrations of cytokines and proteins were decreased at two assessed time points, around 12-24 hours and after 1-2 months. After two months, IL-6 levels decreased to the normal range, by 1.7 -fold, 1.64-fold and 1.62- fold respectively, compared to the control group.

Table 3. Hematological index

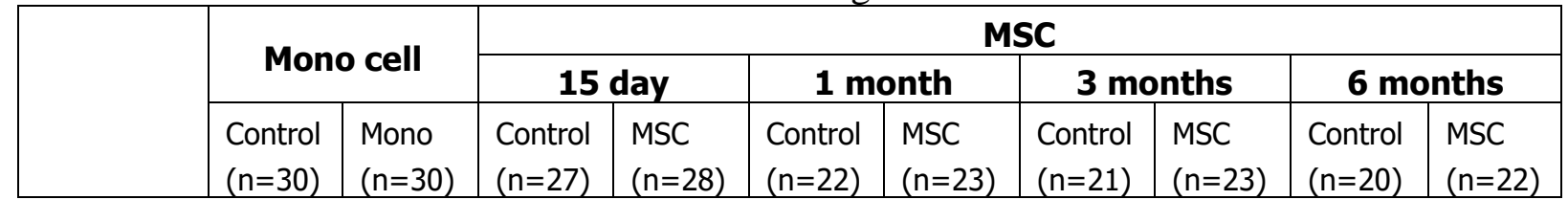


VIETNAM MEDICAL JOURNAL №1\&2/2021

\begin{tabular}{|c|c|c|c|c|c|c|c|c|c|c|}
\hline WBC & $\begin{array}{c} \pm \\
4,40 \\
\end{array}$ & $\begin{array}{c}12,90 \\
\pm 3,93\end{array}$ & $\begin{array}{r}13,66 \\
\pm 3,72\end{array}$ & $\begin{array}{c}12,20 \\
\pm 3,44\end{array}$ & $\begin{array}{c}13,06 \\
\pm 3,10\end{array}$ & $\begin{array}{l}12,25 \\
\pm 2,37\end{array}$ & $\begin{array}{c}12,20 \\
\pm 2,30\end{array}$ & $\begin{array}{c}10,25 \\
\pm 1,94\end{array}$ & $\begin{array}{c}11,40 \\
\pm 2,80\end{array}$ & $\begin{array}{c}11,80 \\
\pm \\
2,09\end{array}$ \\
\hline RBC & $\begin{array}{c}3,27 \\
\pm \\
0,39 \\
\end{array}$ & $\begin{array}{c}3,24 \\
\pm 0,55\end{array}$ & $\begin{array}{c}3,66 \\
\pm 0,28\end{array}$ & $\begin{array}{c}3,79 \\
\pm 0,45\end{array}$ & $\begin{array}{c}3,80 \\
\pm 0,25\end{array}$ & $\begin{array}{c}3,93 \\
\pm 0,40\end{array}$ & $\begin{array}{c}3,85 \\
\pm 0,16\end{array}$ & $\begin{array}{c}3,96 \\
\pm 0,25\end{array}$ & $\begin{array}{c}3,80 \\
\pm 0,37\end{array}$ & $\begin{array}{c}3,84 \\
\pm \\
0,65 \\
\end{array}$ \\
\hline Platelet & $\begin{array}{c}0 \\
\pm \\
69,44\end{array}$ & $\begin{array}{c}0 \\
\pm 78,1 \\
0\end{array}$ & $\begin{array}{l}0 \\
\pm\end{array}$ & $\begin{array}{c}8 \\
\pm\end{array}$ & $\begin{array}{l}2 \\
\pm\end{array}$ & $\begin{array}{l}4 \\
\pm\end{array}$ & $\begin{array}{c}8 \\
\pm \\
52,90\end{array}$ & $\begin{array}{c}165,7 \\
4 \\
\pm \\
65,77\end{array}$ & $\begin{array}{c}174,41 \\
\pm \\
55,00\end{array}$ & $\begin{array}{c}183,2 \\
2 \\
\pm \\
70,44\end{array}$ \\
\hline $\mathrm{Hb}$ & $\begin{array}{c}10,40 \\
\pm \\
1,56 \\
\end{array}$ & $\begin{array}{c}10,30 \\
\pm 1,87\end{array}$ & $\begin{array}{c}10,55 \\
\pm 1,14\end{array}$ & $\begin{array}{c}10,66 \\
\pm 1.70\end{array}$ & $\begin{array}{c}12,07 \\
\pm 1,62\end{array}$ & $\begin{array}{c}12,77 \\
\pm 1,13\end{array}$ & $\begin{array}{c}12,44 \\
\pm 1,51\end{array}$ & $\begin{array}{c}12,98 \\
\pm 2,02\end{array}$ & $\begin{array}{c}12,27 \\
\pm 1,85\end{array}$ & $\begin{array}{c}13,08 \\
\pm \\
1,78 \\
\end{array}$ \\
\hline $\begin{array}{l}\text { Prothromb } \\
\text { in time }\end{array}$ & $\begin{array}{c}13,14 \\
\pm \\
4,32\end{array}$ & $\begin{array}{c}12,80 \\
\pm 3,66\end{array}$ & $\begin{array}{c}12,71 \\
\pm 2,32\end{array}$ & $\begin{array}{c}12,40 \\
\pm 3,10\end{array}$ & $\begin{array}{c}12,50 \\
\pm 2,11\end{array}$ & $\begin{array}{l}12,39 \\
\pm 3,25\end{array}$ & $\begin{array}{c}12,40 \\
\pm 2,00\end{array}$ & $\begin{array}{l}12,30 \\
\pm 2,14\end{array}$ & $\begin{array}{c}12,80 \\
\pm 2,34\end{array}$ & $\begin{array}{c}12,3 \\
\pm \\
2,02\end{array}$ \\
\hline APTT & $\begin{array}{c}36,06 \\
\pm \\
4,83 \\
\end{array}$ & $\begin{array}{c}37,00 \\
\pm 3,97\end{array}$ & $\begin{array}{r}36,00 \\
\pm 2,18\end{array}$ & $\begin{array}{c}38,07 \\
\pm 2,33\end{array}$ & $\begin{array}{c}36,08 \\
\pm 2,10\end{array}$ & $\begin{array}{c}38,14 \\
\pm 2,16\end{array}$ & $\begin{array}{c}36,11 \\
\pm 2,00\end{array}$ & $\begin{array}{r}38,44 \\
\pm 2,45\end{array}$ & $\begin{array}{c}37,16 \\
\pm 1,76\end{array}$ & $\begin{array}{c}38,40 \\
\pm \\
2,45 \\
\end{array}$ \\
\hline $\mathrm{Fi}$ & $\begin{array}{c}3,48 \\
\pm \\
0,22 \\
\end{array}$ & $\begin{array}{c}3,55 \\
\pm 0,18\end{array}$ & $\begin{array}{c}3,15 \\
\pm 0,21\end{array}$ & $\begin{array}{c}3,41 \\
\pm 0,10\end{array}$ & $\begin{array}{c}3,19 \\
\pm 0,25\end{array}$ & $\begin{array}{c}3,52 \\
\pm 0,12\end{array}$ & $\begin{array}{c}3,14 \\
\pm 0,33\end{array}$ & $\begin{array}{c}3,46 \\
\pm 0,18\end{array}$ & $\begin{array}{c}3,26 \\
\pm 0,45\end{array}$ & $\begin{array}{c}3,46 \\
\pm \\
0,24 \\
\end{array}$ \\
\hline
\end{tabular}

Table 4. Respiratory index

\begin{tabular}{|c|c|c|c|c|c|c|c|c|c|c|}
\hline & \multirow{2}{*}{\multicolumn{2}{|c|}{ Mono cell }} & \multicolumn{8}{|c|}{ MSC } \\
\hline & & & \multicolumn{2}{|c|}{15 day } & \multicolumn{2}{|c|}{1 month } & \multicolumn{2}{|c|}{3 months } & \multicolumn{2}{|c|}{6 months } \\
\hline & $\begin{array}{l}\text { Control } \\
(n=30)\end{array}$ & $\begin{array}{l}\text { Mono } \\
(\mathrm{n}=30)\end{array}$ & $\begin{array}{l}\text { Control } \\
(\mathrm{n}=27)\end{array}$ & $\begin{array}{l}\text { MSC } \\
(n=28)\end{array}$ & $\begin{array}{l}\text { Control } \\
(n=22)\end{array}$ & $\begin{array}{l}\text { MSC } \\
(n=23)\end{array}$ & $\begin{array}{l}\text { Control } \\
(n=21)\end{array}$ & $\begin{array}{l}\text { MSC } \\
(n=23)\end{array}$ & $\begin{array}{l}\text { Control } \\
(n=20)\end{array}$ & $\begin{array}{l}\text { MSC } \\
(n=22)\end{array}$ \\
\hline $\mathrm{pH}$ & $\begin{array}{c}7,22 \\
\pm 1,49\end{array}$ & 7,40 & $\begin{array}{c}7,30 \\
\pm 1,41\end{array}$ & $\begin{array}{c}7,38 \\
\pm 0,05\end{array}$ & $\begin{array}{c}7,32 \\
\pm 1,36\end{array}$ & $\begin{array}{c}7,37 \\
\pm 0,16\end{array}$ & $\begin{array}{c}7,42 \\
\pm 1,21\end{array}$ & $\begin{array}{c}7,39 \\
\pm 1,68\end{array}$ & $\begin{array}{c}7,47 \\
\pm 1,15\end{array}$ & $\begin{array}{r}7,35 \\
\pm 1,37\end{array}$ \\
\hline pO2 & $\begin{array}{c}133,21 \\
\pm \\
47,08\end{array}$ & $\begin{array}{c}122,12 \\
\pm 36,0\end{array}$ & $\begin{array}{c}136,01 \\
\pm \\
44,12\end{array}$ & $\begin{array}{l}120,22 \\
\pm 33,08\end{array}$ & $\begin{array}{c}120,00 \\
\pm \\
31,20\end{array}$ & $\begin{array}{c}130,00 \\
\pm \\
13,12 \\
\end{array}$ & $\begin{array}{c}128,13 \\
\pm \\
26,25\end{array}$ & $\begin{array}{c}124,11 \\
\pm \\
10,10\end{array}$ & $\begin{array}{c}76,62 \\
\pm \\
12,44 \\
\end{array}$ & $\begin{array}{c}85,18 \\
\pm \\
11,33\end{array}$ \\
\hline $\mathrm{pCO} 2$ & $\begin{array}{c}41,1 \\
\pm 8,51\end{array}$ & $\begin{array}{c}37,77 \\
\pm 7,14\end{array}$ & $\begin{array}{c}40,22 \\
\pm 6,44\end{array}$ & $\begin{array}{r}37,47 \\
\pm 6,12 \\
\end{array}$ & $\begin{array}{r}40,15 \\
\pm 4,68\end{array}$ & $\begin{array}{c}37,87 \\
\pm 5,40\end{array}$ & $\begin{array}{c}40,22 \\
\pm 4,37\end{array}$ & $\begin{array}{l}37,80 \\
\pm 5,24\end{array}$ & $\begin{array}{c}40,33 \\
\pm 5,14\end{array}$ & $\begin{array}{r}38,20 \\
\pm 5,47\end{array}$ \\
\hline $\mathrm{HCO}^{-}$ & $\begin{array}{c}22,22 \\
\pm 3,90\end{array}$ & $\begin{array}{c}24,08 \\
\pm 3,02\end{array}$ & $\begin{array}{c}21,27 \\
\pm 3,33\end{array}$ & $\begin{array}{r}23,05 \\
\pm 3,16\end{array}$ & $\begin{array}{c}21,2 \\
\pm \\
3,60\end{array}$ & $\begin{array}{c}23,15 \\
\pm 4,04\end{array}$ & $\begin{array}{c}21,24 \\
\pm 3,70\end{array}$ & $\begin{array}{l}23,19 \\
\pm 3,50\end{array}$ & $\begin{array}{c}22,18 \\
\pm 3,22\end{array}$ & $\begin{array}{c}23,44 \\
\pm 3,13\end{array}$ \\
\hline $\mathrm{SaO} 2$ & $\begin{array}{l}89,22 \\
\pm 6,78 \\
\end{array}$ & $\begin{array}{l}89,22 \\
\pm 6,78 \\
\end{array}$ & $\begin{array}{l}89,70 \\
\pm 6,44 \\
\end{array}$ & $\begin{array}{r}88,27 \\
\pm 6,40 \\
\end{array}$ & $\begin{array}{l}89,78 \\
\pm 6,00 \\
\end{array}$ & $\begin{array}{r}88,38 \\
\pm 6,12 \\
\end{array}$ & $\begin{array}{l}88,55 \\
\pm 5,97 \\
\end{array}$ & $\begin{array}{l}90,31 \\
\pm 7,22 \\
\end{array}$ & $\begin{array}{r}88,45 \\
\pm 5,77 \\
\end{array}$ & $\begin{array}{l}91,22 \\
\pm 3,24\end{array}$ \\
\hline $\mathrm{Na}$ & $\begin{array}{c}135,86 \\
\pm 6,44\end{array}$ & $\begin{array}{c}132,60 \\
\pm 6,78\end{array}$ & $\begin{array}{c}134,41 \\
\pm 6,20\end{array}$ & $\begin{array}{r}135,60 \\
\pm 6,01\end{array}$ & $\begin{array}{c}135,25 \\
\pm 7,66\end{array}$ & $\begin{array}{c}137,20 \\
\pm 5,31\end{array}$ & $\begin{array}{c}138,11 \\
\pm 6,55\end{array}$ & $\begin{array}{c}139,40 \\
\pm 5,00\end{array}$ & $\begin{array}{c}135,87 \\
\pm 5,05\end{array}$ & $\begin{array}{r}137,16 \\
\pm 4,65\end{array}$ \\
\hline K & $\begin{array}{c}4,33 \\
\pm 1,00\end{array}$ & $\begin{array}{c}3,80 \\
\pm 2,05\end{array}$ & $\begin{array}{c}4,35 \\
\pm 1,12\end{array}$ & $\begin{array}{c}3,87 \\
\pm 1,35\end{array}$ & $\begin{array}{c}4,31 \\
\pm 1,05\end{array}$ & $\begin{array}{c}3,64 \\
\pm 1,30\end{array}$ & $\begin{array}{c}4,49 \\
\pm 1,12\end{array}$ & $\begin{array}{c}3,52 \\
\pm 1,21\end{array}$ & $\begin{array}{c}4,50 \\
\pm 1,17\end{array}$ & $\begin{array}{c}4,05 \\
\pm 0,51\end{array}$ \\
\hline
\end{tabular}


The results of the subclinical assessment of patients after MNC and MSC transplantation at the dose of $6-9 \times 10^{6}$ cells/kg cells showed liver, kidney, cortisol, lactate, glucose, hematologic indices, and respiratory indices. No difference compared with the control group. The respiratory index of transplanted patients did not change, indicating no pulmonary embolism. Frederic Baron et al. showed that MSC injection appeared to be safe, with no infusion toxicity (and in particular no reports of pulmonary embolism) [9]. The stability of hematological indices reflects the safety of MSC transplantation in patients. Guo et al. showed that MSCs have the ability to regulate the endogenous microenvironment and "immune privilege" that allows them to escape attack by the immune system [10]. Therefore, there is no shock or allergic reaction when transplanting stem cells. In contrast, the inflammatory index IL-6 had a marked change between the 2 study groups. The IL- 6 index in the MSC transplant group (354.25 \pm 215.46 to $31.21 \pm 8.33$ ) was reduced 11.35 times after 6 months of treatment and 2.25 times higher than the control group $(70.33 \pm$ 10.37). Student t-tests calculate the significant difference between two groups, $* * \mathrm{P}<0.01 ; * \mathrm{P}<0.05$

\section{DISCUSSION}

Similar studies have demonstrated, Tian et al. showed that 38 of 97 patients (39.2\%) had improved brain function after mesenchymal stem cell transplantation from the marrow, and found no evidence of toxicity [6]. Similarly, Cox et al. injecting MNBMSC into 15 patients with severe traumatic brain injury showed no serious side effects related to cell injection. Although the treatment group had more severe lesions, there was structural preservation of important regions of interest correlated with functional outcome [7]. However, the sample size of this study was too small, and the time interval between MCS implantation and injury of direct and intravenous infusion was different. Furthermore, the number of MSCs injected into each patient was variable, and no subclinical and clinical analyzes of the combined effects of MSCs on the lesion site were recorded. This study aimed to evaluate the safety and feasibility of stem cell transplantation through clinical and laboratory parameters in TBI patients. The efficacy of the therapy can be traced back to the transplantation of MNCs and MSCs at a dose of $9 \times 10^{6}$ cells $/ \mathrm{kg}$ cells, which combine to facilitate the functional efficiency of stem cells. MNC transplantation is performed urgently for approximately $24 \mathrm{~h}$ to reduce the risk of acute inflammation induced by the first waves of cytokines and innate immune cells [11]. Cox et al. demonstrated the feasibility of MNCs in TBI, with no patients presenting with severe symptoms of cell transplantation [7]. We also performed the second engraftment with MSCs after culture to improve the low number of first engraftments, reduce chronic inflammation, and improve the microenvironment thanks to the immunoregulatory function of MSCs. The quality of MNC and MSC was assured through various assessments such as FACS, CFU-F, and differentiation. We performed transplants with low cell counts $\left(6-9 \times 10^{6}\right.$ cells $/ \mathrm{kg}$ ) to reduce the risk of cell accumulation in the lungs. Results revealed no evidence of venous thrombosis, tumor formation, intracranial infection, or systemic infection in any of the patients at the first 
transplant and after the 6-month evaluation (Table3, Table 4).

The subclinical (liver index, kidney, cortisol, lactate, glucose, hematological index, and respiratory index) and clinical (pulse, temperature, SBP, HATtr, HATTB) indices on 2 study groups are not significantly different. A total of 18 deaths (Control: 10 and MSC: 8) were due to disease progression, not caused by complications of MSCs. The patients did not find pulmonary venous occlusion or coagulation due to cell transplantation. The safety and efficacy and absence of adverse effects of pluripotent stem cells have been observed in many clinical trials such as grafthost disease and systemic lupus erythematosus [12], [13]. Our results are similar to those of Tian et al. showed that the use of MSCs had a significant effect after TBI [6]. A total of 97 patients (24 with persistent vegetative state and 73 with dyskinesia) were treated with autologous bone marrow mesenchymal stem cell therapy. 38 out of 97 patients $(39.2 \%)$ had improved brain function after transplantation. 11 out of 24 patients $(45.8 \%)$ with persistent vegetative states showed post-treatment improvement in consciousness. Twentyseven out of 73 patients $(37.0 \%)$ with dyskinesia began to show improvement in motor functions. None of the cases showed shock or complications from MSC transplantation.

GCS and IL-6 indexes were significantly changed between the cell transplant group and the control group after 6 months of treatment. After 1 month in the stem cell transplant group, the GCS index was increased by 1.12 times than the control group (Control: $6.7 \pm 0.89$; MSC: $7.5 \pm 1.65$ ), after 6 months GCS index of the MSC transplant group was $8.78 \pm 1.09$. According to Mehta R et al., the GCS score of 9-12 is classified as moderate trauma, showing that the patients with TBI have recovered from the critical condition. In contrast, the GCS index of the control group after 6 months is still below $9 \quad(7.34 \pm 1.61)$ [8]. For the inflammatory index IL-6, in the MSC transplant group $(354.25 \pm 215.46$ to $31.21 \pm$ 8.33 ), there was a reduction of 11.35 times after 6 months of treatment and 2.25 times higher than that of the control group $(70.33 \pm$ 10.37).

Our results are similar to those of Cox et al.,10 children with TBI trauma with a GCSscore of 5 to 8 were transplanted with MSCs and followed up for 6 months [14]. Inflammatory reactions following TBI often lead to multi-organ dysfunction or even death [15]. Various studies have also reported increased and elevated levels of inflammatory molecules in the blood after brain injury, including CRP, TNF- $\alpha$, ROS, IL-6, and others. [16]. Thus, decreased IL-6 levels in the MSC transplant group contributed to a decrease in systemic candidate responses, suggesting a strong induction between MSCs and TBI-derived immune responses that reduce the risk of exacerbation. Additional lesions (Figure 4C). Chang YS et al. performed endotracheal MSC transplantation in 9 children with bronchopulmonary dysplasia (BPD) that showed levels of interleukin-6, interleukin-8, substrate metalloproteinase-9, TNF- $\alpha$, and transforming growth factor $\beta 1$ during puncture Tracheal suction at day 7 was significantly reduced compared with baseline [17].

In conclusion, 22/30 severe TBI patients were successfully treated by MNC and MSC transplantation with a graft dose of $6-9 \times 10^{6}$ 
cells $/ \mathrm{kg}$. All patients after transplantation did not find any complications, showing that the graft dose of $9 \times 10^{6}$ cells $/ \mathrm{kg}$ is feasible and safe in the treatment of TBI. The paraclinical and clinical indices were stable between the control group and the MSC group. The IL-6 and GCS indices reflect the antiinflammatory effects of MSCs to help patients improve their health status.

\section{CONCLUSION}

- There was no difference in clinical and laboratory parameters between the 2 study groups. MSC transfusions are safe and may serve as a novel therapeutic approach for TBI patients.T

- With MNC and MSC grafting dose of $9 \times 10^{6}$ cells $/ \mathrm{kg}$ safe for the patient's body, no complications were detected after transplantation as well as after 6 months of treatment.

- The IL-6 index in the MSC transplant group was lower than that in the control group, suggesting that MSCs have the ability to regulate inflammation after TBI by reducing the expression level of IL-6. This may lead to a better improvement in the GCS index of the MSC transplant group compared to the control group

\section{ACKNOWLEDGEMENTS}

This research was supported by a grant from Ministry of Science \& Technology Viet Nam.

\section{REFERENCES}

1. Gardner RC, Burke JF, Nettiksimmons J, Kaup A, Barnes DE, Yaffe K. Dementia risk after traumatic brain injury vs nonbrain trauma: the role of age and severity. JAMA Neurol. 2014;71:14907.10.1001/jamaneurol.2014.2668.
2. Von Leden R.E., Yauger Y.J., Khayrullina G., Byrnes K.R. Central nervous system injury and nicotinamide adenine dinucleotide phosphate oxidase: oxidative stress and therapeutic

Neurotrauma. 2017;34:755-764.

3. Sanchez-Ramos J., Song S., CardozoPelaez F., et al. Adult bone marrow stromal cells differentiate into neural cells in vitro. Experimental

Neurology. 2000;164:247-256. doi: 10.1006/exnr.2000.7389.

4. Borlongan CV. Bone marrow stem cell mobilization in stroke: a 'bonehead' may be good after all! Leukemia. 2011;25: 1674-1686

5. Walker PA, Shah SK, Harting MT, Cox CS. Progenitor cell therapies for traumatic brain injury: barriers and opportunities in translation. Dis Model Mech. 2009;2:2338.10.1242/dmm.001198.

6. Tian $C$, Wang $X$, Wang $X$, Wang $L$, Wang $\mathbf{X}$, Wu S, Wan Z. Autologous bone marrow mesenchymal stem cell therapy in the subacute stage of traumatic brain injury by lumbar puncture. Exp Clin Transplant. 2013 Apr 1;11(2):176-81.

7. Cox Jr CS, Hetz RA, Liao GP, Aertker BM, Ewing-Cobbs L, Juranek J, Savitz SI, Jackson ML, Romanowska-Pawliczek AM, Triolo F, Dash PK. Treatment of severe adult traumatic brain injury using bone marrow mononuclear cells. Stem Cells. 2017 Apr;35(4):1065-79.

8. Mehta R, Chinthapalli K. Glasgow coma scale explained. BMJ. 2019 May 2;365.

9. Baron F, Storb R. Mesenchymal stromal cells: a new tool against graft-versus-host disease?. Biology of Blood and Marrow Transplantation. 2012 Jun 1;18(6):822-40.

10. Guo J, Huang Z, Lin L, Lv J. Coronavirus disease 2019 (COVID-19) and cardiovascular disease: a viewpoint on the potential influence of angiotensin-converting enzyme inhibitors/angiotensin receptor blockers on onset and severity of severe acute respiratory syndrome coronavirus 2 infection. Journal of the American Heart Association. 2020 Apr 9;9(7):e016219. 
11. Vaibhav $K$, Braun $M$, Alverson $K$, Khodadadi H, Kutiyanawalla A, Ward A, Banerjee C, Sparks T, Malik A, Rashid MH, Khan MB. Neutrophil extracellular traps exacerbate neurological deficits after traumatic brain injury. Science advances. 2020 May 1;6(22):eaax8847.

12. Hashmi S, Ahmed M, Murad MH, Litzow MR, Adams RH, Ball LM, Prasad VK, Kebriaei P, Ringden O. Survival after mesenchymal stromal cell therapy in steroidrefractory acute graft-versus-host disease: systematic review and meta-analysis. The Lancet Haematology. 2016 Jan 1;3(1):e45-52.

13. Kamen DL, Nietert PJ, Wang H, Duke T, Cloud C, Robinson A, Gilkeson GS. CT-04 Safety and efficacy of allogeneic umbilical cord-derived mesenchymal stem cells (MSCs) in patients with systemic lupus erythematosus: results of an open-label phase I study.

14. Cox Jr CS, Baumgartner JE, Harting MT, Worth LL, Walker PA, Shah SK, EwingCobbs L, Hasan KM, Day MC, Lee D,
Jimenez F. Autologous bone marrow mononuclear cell therapy for severe traumatic brain injury in children. Neurosurgery. 2011 Mar 1;68(3):588-600.

15. Clark RS, Schiding JK, Kaczorowski SL, Marion DW, Kochanek PM. Neutrophil accumulation after traumatic brain injury in rats: comparison of weight drop and controlled cortical impact models. Journal of neurotrauma. 1994 Oct;11(5):499-506.

16. Venetsanou $K$, Vlachos $K$, Moles $A$, Fragakis G, Fildissis G, Baltopoulos G. Hypolipoproteinemia and hyperinflammatory cytokines in serum of severe and moderate traumatic brain injury (TBI) patients. European cytokine network. 2007 Oct 26;18(4):39-42.

17. Chang YS, Ahn SY, Yoo HS, Sung SI, Choi SJ, Oh WI, Park WS. Mesenchymal stem cells for bronchopulmonary dysplasia: phase 1 dose-escalation clinical trial. The Journal of pediatrics. 2014 May 1;164(5):966-72. 